\title{
Mixed Vehicular Traffic Flow Model on an Inclined Multilane Road
}

\author{
Delina Mshai Mwalimo $^{1 *}$, Mary Wainaina ${ }^{1}$, Winnie Kaluki ${ }^{1}$. \\ ${ }_{1 *, 1,1}$ Department of Mathematics and Actuarial Science, \\ Catholic University of Eastern Africa, Box 62157-00200, Nairobi-Kenya
}

\begin{abstract}
This study outlines the Kerner's 3 phase traffic flow theory, which states that traffic flow occurs in three phases and these are free flow, synchronized flow and wide moving jam phase. A macroscopic traffic model that is factoring road inclination is developed and its features discussed. By construction of the solution to the Rienmann problem, the model is written in conservative form and solved numerically. Using the Lax-Friedrichs method and going ahead to simulate traffic flow on an inclined multi lane road. The dynamics of traffic flow involving cars(fast moving) and trucks(slow moving) on a multi-lane inclined road is studied. Generally, trucks move slower than cars and their speed is significantly reduced when they are moving uphill on an in- clined road, which leads to emergence of a moving bottleneck. If the inclined road is multi-lane then the cars will tend to change lanes with the aim of overtaking the slow moving bottleneck to achieve free flow. The moving bottleneck and lanechange ma- noeuvres affect the dynamics of flow of traffic on the multi-lane road, leading to traffic phase transitions between free flow (F) and synchronised flow(S). Therefore, in order to adequately describe this kind of traffic flow, a model should incorporate the effect of road inclination. This study proposes to account for the road inclination through the fundamental diagram, which relates traffic flow rate to traffic density and ultimately through the anticipation term in the velocity dynamics equation of macroscopic traffic flow model.
\end{abstract}

The features of this model shows how the moving bottleneck and an incline multilane road affects traffic transistions from Free flow(F) to $\operatorname{Synchronised~flow(S).~}$ For a better traffic management and control, proper understanding of traffic congestion is needed. This will help road designers and traffic engineers to verify whether traffic properties and characteristics such as speed(velocity), density and flow among others determines the effectiveness of traffic flow.

Keywords:- Fundamental Diagram, Moving bottleneck, Phase transitions, Traffic flow, Three phase traffic flow.

\section{INTRODUCTION}

Transport is a key element of generating an aggressive bussiness domain and also forms a way in which the nation can achieve its environmental objectives, social and economic goals. Peoples' quality of life has largely been affected by congestion of traffic of vehicles. This is due to accidents, environmental pollution and also delays. A well systematic transport structure that reduces external influences and travel time should be considered. Increasing the exisisting roadways capacity, is one way of getting rid of the traffic congestion in our roads, this can be done by constructing by-passes, transport corridors as well as surface streets. In most cases, however, this is impossible because of local politics, lack of financial resources, lack of enough space and environmental hazards.

In real world, the highways are never horizontal. There exist various highway sections with gradients either as an incline (uphill) or decline (downhill). These road conditions significantly affect the dynamics of traffic that is often comprised of cars and trucks since drivers continuously adjust their vehicle speeds in response to prevailing road gradient and the density of vehicles ahead. The slow moving of trucks uphill tends to impede the free flow of fast cars on the road, as a result congestion starts to build up.

Trucks moving slowly form a moving bottleneck in vehicular traffic on a multi-lane road. A moving bottleneck causes disruption of traffic flow such as capacity drop due to the traffic jam. Though in some highways climbing lane in the uphill segments have been built, however,there still some highways where this is not done probably due to due to the high cost involved in construction of such. Traffic flow modelling and simulation is a cost effective way to evaluate the performance of the designed uphill road segment.

A large amount of experimental research has been done on traffic flow theories and traffic congestion. The wave theory of kinematics, classical flow congestion, LWR traffic flows congestion theory by[11] were among the earliest well persuasive theories of traffic flow. Three areas of fundamental identity of traffic flow theory were used in this theory. According to the theory the flow has been obtained by the equation of continuity for a fluid (conservation of mass), multiplying velocity with density, and a term showing the relationship between density and 
velocity. This has shown a number of serious deficiencies being a first order approximate model.

Equation of motion of a car following theory, given by, response $=$ sensitivity $\times$ stimulus, was derived by[5] to try to overcome the LWR model deficiencies. This model was known as PW models. The Payne's model combined with its computer applications brought forth many interests in higher order in a continuous non spatial traffic flow models, but the application of this model reported varied results. Higher order terms were then neglected by[6] when he was deriving the new theory.

This made [1]to be concerned as to whether higher order terms can be abandoned when the traffic concentration on the road was changing fast, as occurs near a region of disturbance as in shocks. He concluded that these higher order terms can be abandoned if the concentration function $\rho(x, t)$ is deferential and time-space related scales are well handled. This led Aw Rascle as discussed by [2] to come up with a model which would correct the above inconsistencies. All the natural requirements (frontal stimuli) were obeyed by the AwRascle model. The inconsistencies of 'wrong way travel' of second order models disappeared.

A macroscopic traffic flow model from a macroscopic follow the leader models were derived by [3]. This model obtained by Günther et al. [3] was of Aw-Rascle design. Several researchers have studied flow of traffic on uphill gradients. Some focussed on the contribution of the uphill gradient in formation of stop-and-go traffic jams[12], while others focussed on the effect of the positive slope on speed of vehicles due to gravitational force[10]. They all established that traffic jam usually arises on inclined roads,either due to car following each other which results into stop-and-go jam or traffic jam that is influenced by gravitational force that is acting on the vehicles.

None of existing literature have sought to explain the impact of uphill gradients on vehicles' speed on a multilane road through the fundamental diagram based on[7] three phase theory by distinguishing the traffic as fast moving (cars) and slow mov- ing (trucks) whose traffic states lie on different locations in the fundamental diagram. This forms the aim of this study and whose possible impact is the improvement of existing macroscopic models for multilane mixed traffic flows to better account for the traffic nonhomogeneity and road gradients.

\section{MODEL FORMULATION}

The equations governing flow in traffic flow are based on the momentum and conser- vation of mass. The mathematical modelling of traffic flow often rests on a fluid flow analogy, treating the traffic stream as a one dimensional compressible fluid from which it can be deduced that traffic flow is conserved. The number of vehicles entering a cer- tain region equals the number leaving the same region, with an assumption that there are no exits, entrances or breakdown of vehicles within the region.

The numerical solution to the system was achieved by use of numerical scheme based on the Lax-Friedrichs method. This scheme is a conservative numerical scheme for solving partial differential equations. A computer software MATLAB is also be used. The results of traffic density, velocity and flow rate is depicted using the Fundamental Diagrams that is based on Kerner's three phase theory. In addition the relationship of traffic density, velocity and flow rate is discussed with regard to the road inclination. A model is developed for mixed traffic flow on an inclined road by solving the partial differential equation of continuity for both trucks (slow moving) and cars (fast mov- ing). A velocity dynamic equation for cars is solved. This equation is used to describe time varying and spatially varying average velocities $\mathrm{u}(\mathrm{x}, \mathrm{t})$, such as those that occur in traffic jams or stop and go jams [4]

The velocity for cars is considered to be dynamic while that of trucks is considered to be a low constant speed. The velocity of both truck depends on the density of the trucks $\rho(T)$ and by an angle, $\alpha$, of the road inclination, while that of a car depends on the density of the trucks $\rho(T)$. The bottleneck flow is caused by the slow moving trucks.

\section{GOVERNING EQUATIONS FOR TRUCKS}

A conserved quantity cannot increase or decrease, it can only move from place to place. In traffic flow, the number of vehicles is conserved, assuming there are no exits or en- trance or breakdowns of vehicles, then the number of vehicles entering a certain region equals the number leaving the same region, hence we use the equation of continuity. The motion of trucks on roads can be adequately described by the LWR model, which usually comprises of the equation of conservation of trucks and a constitutive relation to express the velocity as a function of the trucks density. This equation expresses the relation between the rates of change of the truck's density with respect to time (t) and their flow rate with respect to space $(\mathrm{x})$. 


$$
\frac{\partial \rho_{T}}{\partial t}+\frac{\partial \rho v_{T}\left(\rho_{T}\right)}{\partial x}=0
$$

Where $\rho_{\mathrm{T}}$ is the density of the trucks and is $\mathrm{v}_{\mathrm{T}}$ the equilibrium or optimal velocity of the truck and $\mathrm{v}_{\mathrm{T}}$ is given by the relation;[9]

$$
v(\rho)=v_{\max } \tanh \left[\frac{\frac{1}{\rho}-h_{o}}{c_{o} v_{\max }}\right]
$$

Where $h_{0}$ denote an average space gap on the highway and $c_{o}$ is a shape parameter. The existence of a road inclination for vehicular traffic comprised of cars and trucks often results to the vehicles moving uphill with minimal space gap between them.

\section{GOVERNING EQUATIONS FOR CARS}

The motion of cars on roads can be appropriately described by the Aw-Rascle model, which comprises of the equation of conservation of cars and an equation describing the velocity dynamics.

$$
\frac{\partial \rho_{c}}{\partial t}+\frac{\partial \rho_{c} u_{c}}{\partial x}=0
$$

Where $\rho c$ is the density of the cars and uc the velocity of the car. The equation ex- presses the relation between the rates of change of density of cars with respect to time and flow with respect to space, and the dynamic velocity equation given by;

$$
\frac{\partial \rho_{c} u_{c}}{\partial t}+\frac{\partial\left(\rho_{c} u_{c}^{2}\right)}{\partial x}-\rho_{c}^{2} P^{\prime}\left(\rho_{c}\right) \frac{\partial u}{\partial x}=0
$$

The above quation is used to describe time varying and spatially varying average ve- locities $u(x, t)$ such as those that occur in traffic jams or stop and go jams [4] where the relation[9] is as follows.

$$
P^{\prime}\left(\rho_{c}\right)=\beta\left[\frac{\rho_{c}}{1-\rho_{c}}\right]
$$

The term $\beta\left[\frac{\rho_{c}}{1-\rho_{c}}\right] \frac{\partial u_{c}}{\partial x}$ is the anticipation term. The variable $\beta$ is a measure of road inclination and in this study we shall approximate it as a function of velocity as follows;

$$
\beta=A e^{-u_{c}}
$$

where $\mathrm{A}$ is a contant and $\mathrm{u}_{\mathrm{c}}$ is the dynamic velocity of the cars in the moving bottle neck.

The [8] study indicate that the existence of a road inclination (uphill gradient) in vehic- ular traffic comprised of cars and trucks often results to the vehicles moving uphill with minimal space gap between them. This leads to a platoon effect and hence a moving bottleneck ensues. Therefore we can account for the road inclination as in eq. (4.5) by replacing the constant $h_{0}$ in (3.2) with a variable one $h=h_{0}-\alpha$, whereby the angle of road inclination $0 \leq \alpha \leq \Pi 4$. The relation $h=h_{0}-\alpha$ shows that the average space gap reduces uphill.

$$
V^{M}(\rho, \alpha)=V_{\max }^{M} \tanh \left[\frac{\frac{1}{\rho}-h}{C_{o} V_{\max }^{M}}\right]
$$

where $\mathrm{V}^{\mathrm{M}}{ }_{\text {max }}$ denote maximum velocity of the moving bottleneck, and dictated by the leading truck on the uphill gradient. usually $\mathrm{V}_{\text {max }}^{\mathrm{M}}<\mathrm{uc}$ since trucks have less acceler- ation ability as compared to cars. For $\alpha=0$,equations (4.5) reduces to (3.2) but 
for non-zero values the average space gap tends to decrease in a multilane road, unlike in one way road. This is because the cars will change-lanes to overtake the slow moving bottleneck.

The presence of the road inclination contributes to the formation of congested traffic. Therefore it is expected to influence the traffic anticipation term that is present in the velocity dynamics equation of macroscopic traffic flow models[9] .

$$
\frac{\partial \rho_{c} u_{c}}{\partial t}+\frac{\partial\left(\rho_{c} u_{c}^{2}\right)}{\partial x}-\beta\left[\frac{\rho_{c}}{1-\rho_{c}}\right] \frac{\partial u_{c}}{\partial x}=0
$$

where $\mathrm{u}$ is the traffic average velocity and $\beta$ is a constant that significantly influences the formation and dissolution of congested traffic patterns during model simulation. $\beta\left[\frac{\rho_{c}}{1-\rho_{c}}\right] \frac{\partial u_{c}}{\partial x}$ this is the anticipation term, it describes the longitudinal interaction of vehicles moving in the same direction on the road. These interactions involve braking and acceleration of vehicles in response to the frontal stimuli. Therefore in essence the anticipation term describes the formation and dissolution of traffic jams. Since uphill road gradients leads to emergence of moving bottlenecks, this study proposes to im- prove the anticipation term bt replacing the constant $\beta$ by a more dynamic expression;

$$
\beta(\rho, \alpha)=A e^{-u_{c}}
$$

where A is a constant and uc velocity of a car in a moving bottle neck. This paves way for the determination of the effects of a moving bottleneck on the rest of the traffic flow upstream of it.

Substituting for (4.7) in (4.6) above we get;

$$
\frac{\partial \rho_{c} u_{c}}{\partial t}+\frac{\partial\left(\rho_{c} u_{c}^{2}\right)}{\partial x}-A\left[\frac{e^{-\left(u_{c}\right) \rho_{c}}}{1-\rho_{c}}\right] \frac{\partial u_{c}}{\partial x}=0
$$

we can re-write the model equations in non-conseravative form in terms of $\rho$ and $u$ as follows.

$$
\frac{\partial \rho_{c}}{\partial t}+\frac{\partial \rho_{c} u_{c}}{\partial x}=0
$$

using expression (4.3) in (4.8) yields.

$$
\begin{gathered}
\frac{\partial \rho_{c} u_{c}}{\partial t}+\frac{\partial \rho u_{c}^{2}}{\partial x}-\rho_{c}^{2}\left[p^{\prime}\left(\rho_{c}\right)\right] \frac{\partial u}{\partial x}=0 \\
u_{c} \frac{\partial \rho_{c}}{\partial t}+\rho_{c} \frac{\partial u_{c}}{\partial t}+u_{c}^{2} \frac{\partial \rho_{c}}{\partial x}+2 u_{c} \rho_{c} \frac{\partial u_{c}}{\partial x}-\rho_{c}^{2}\left[p^{\prime}\left(\rho_{c}\right)\right] \frac{\partial u}{\partial x}=0 \\
\frac{\partial u_{c}}{\partial t}+\left[u_{c}-e^{-u_{c}} \rho_{c} p^{\prime}(\rho)\right] \frac{\partial u_{c}}{\partial x}=0
\end{gathered}
$$

in order to express the equation in conservative form we multiply (4.9) with $\mathrm{p}^{0}$ to obtain

$$
\begin{aligned}
& p^{\prime} \rho \frac{\partial u_{c}}{\partial x}=-p^{\prime} \frac{\partial \rho_{c}}{\partial t}-p^{\prime} u_{c} \frac{\partial \rho_{c}}{\partial x} \text {. } \\
& e^{u_{c}} \frac{\partial u_{c}}{\partial t}+u_{c} e^{u_{c}} \frac{\partial u_{c}}{\partial x}+p^{\prime} \frac{\partial \rho_{c}}{\partial t}+p^{\prime} u_{c} \frac{\partial \rho_{c}}{\partial x}=0
\end{aligned}
$$

Grouping the expressions gives 
$\partial_{t}\left(e^{u_{c}}+p^{/}\right)+\partial_{x}\left(e^{u_{c}}+p^{/}\right) u_{c}=0$

multiplying (4.9) by $\left(e^{u_{c}}+p^{\prime}\right)$ we get

$$
\left(e^{u_{c}}+p^{\prime}\right) \partial_{t} \rho_{c}+\left(e^{u_{c}}+p^{\prime}\right) \partial_{x} \rho_{c} u_{c}=0
$$

multiplying (4.9) by $\rho_{\mathrm{c}}$ and adding result to (4.11) to get the conservative form after regrouping the terms

$$
\begin{gathered}
\rho_{c} \frac{\partial \rho_{u}}{\partial t}+\rho_{c} \frac{\partial \rho_{c} u_{c}}{\partial x}=0 \\
\partial_{t}\left(\rho_{c}\right)+\rho_{c} \partial_{x}\left(\rho_{c}+u_{c}\right)=0 \\
\partial_{t}\left(e^{u_{c}}+p^{\prime}\right)+u_{c} \partial_{x}\left(e^{u_{c}}+p^{\prime}\right)=0 \\
\partial_{t}\left[\rho_{c}\left(e^{u_{c}}+p^{\prime}\right)\right]+\partial_{x}\left[\rho_{c} u_{c}\left(e^{u_{c}}+p^{\prime}\right)\right]=0 \\
\partial_{t} \rho_{c}+\partial_{x} \rho_{c} u_{c}=0
\end{gathered}
$$

where the consrvative variables are $\rho_{c}$ and $y=\rho_{c} e^{u_{c}}+\rho_{c} p$

At the bottleneck, cars slows down and adopt velocity of slow moving trucks if the can't overtake. Therefore, for a road segment, the initial conditions; $[-1,0,1]$ we have that, at $x=-1$, this is a region before the start of the incline road, where the velocity of the cars $\mathrm{u}_{\mathrm{c}}$ is greater than the velocity of the truck $\mathrm{V}_{\mathrm{T}}$. at $\mathrm{x}=0$, this is the beggining of the incline road, where fast moving cars are forced to follow the slow moving trucks when they fail to overtake, at this point the cars are forced to move with the speed of moving bottleneck, $\mathrm{u}_{\mathrm{c}}=\mathrm{V}$

\section{METHOD OF SOLUTION}

We construct a Finite Difference Scheme (FDS) to find a numerical approximation solution to the conservative system (4.13)and (4.14) will be achieved by use of the numerical scheme based on the Lax-Friedrichs method.

Writing the system of equation in the form ;

$$
U_{t}+F(U)_{x}=0
$$

where the independent variables are $\mathrm{t}(\mathrm{time})$ and $\mathrm{x}$ (space).

where the dependent variable, $U=\left[U_{1}, U_{2}\right]=\left[\begin{array}{l}\rho_{c} \\ u_{c}\end{array}\right]$ and $\mathrm{F}$ is a function of $\mathrm{U}$, where $F(U)=\left[F_{1}, F_{2}\right]=\left[\rho_{c} u_{c}, \rho_{c} y\right]$

and $\mathrm{x}$ is restricted to the finite domain $[-1,1]$

The initial conditions for the cars model are specified as follows;

$$
\begin{gathered}
x=0 ; \rho_{c}(x, 0)=\rho_{T}(x, 0) \text { and } u_{c}(x, 0)=V^{T}(x, 0) \\
x \neq 0 ; \rho_{c}(x, 0)=0.15, u_{c}(x, 0)=V^{c}\left(\rho_{c}(x, 0)\right)
\end{gathered}
$$

and the transmissive Boundary Conditions are as follows; 


$$
\begin{gathered}
U_{0, j}=U_{1, j} \\
U_{N, j}=U_{N-1, j}
\end{gathered}
$$

We will create a FDS to approximate $U$ at a finite set of values of the independent variables. The approximate value of $U$ on this finite set will be denoted by $u$.

Spatial and Time Discretisation.

Fixing t at $t=t_{n}$, we approximate the spatial derivative, $F(U)_{x}$ in (5.1) at each point $\left(x_{i}, t_{n}\right)$ using the forward difference formula.

$$
F(U)_{x}\left(x_{i}, t_{n}\right)=\frac{F\left(U_{i+1}^{n}\right)-F\left(U_{i}^{n}\right)}{\triangle x}
$$

Replacing (5.6) in (5.1) for $F(U)_{x}$ yields;

$$
U_{t}+\frac{F\left(U_{i+1}^{n}\right)-F\left(U_{i}^{n}\right)}{\triangle x}=0
$$

Fixing $\mathrm{x}$ at $\mathrm{x}=x_{i}$, we approximate the partial derivative, $U_{t}$ in (5.1) at each point $\left(x_{i}, t_{n}\right)$ using the first order forward difference, (where $\Delta t$ is the spacing between time interval).

$$
U_{t}=\frac{U_{i}^{n+1}-U_{i}^{n}}{\triangle t}
$$

On substituting (5.8) for $U_{t}$ in (5.1) becomes

$$
\frac{U_{i}^{n+1}-U_{i}^{n}}{\Delta t}+\frac{F\left(U_{i+1}^{n}\right)-F\left(U_{i}^{n}\right)}{\Delta x}=0
$$

Which on re arranging yields;

$$
U_{i}^{n+1}=U_{i}^{n}-\frac{\triangle t}{\triangle x}\left(F\left(U_{i+1}^{n}\right)-F\left(U_{i}^{n}\right)\right)=0
$$

Using Lax-Friedrichs Scheme,based on FTCS( Forward in Time, Centred in Space) the above scheme (5.10)can be rewritten as;

$$
U_{i}^{n+1}=\frac{1}{2}\left(U_{i+1}^{n}+U_{i-1}^{n}\right)-\frac{1}{2} \lambda\left[F\left(U_{i+1}^{n}\right)-F\left(U_{i-1}^{n}\right)\right]
$$

where $\lambda=\frac{\Delta t}{\Delta x}$ is the abbreviation of the ratio of mesh widths, and

$$
U(x, t)=U(i \triangle x, n \triangle t)=U(i, n)=U_{i}^{n}=\left[\begin{array}{l}
\rho_{c} \\
u_{c}
\end{array}\right]
$$


Where the initial conditions are impemented as follows;

$U_{i}^{n}=\left[\begin{array}{c}\rho_{c i}^{n} \\ u_{c}^{n}\end{array}\right]$

Implying that

$$
\left(\rho_{c}\right)_{i}^{0}=\left(\rho_{T}\right)_{i}^{0}
$$

and

$$
\left(u_{c}\right)_{i}^{0}=\left(V^{T}\right)_{i}^{0}
$$

VI. SIMULATION RESULTS: EFFECT OF INCLINATION ON FUNDA-MENTAL DIAGRAM

6.1

$$
\text { For } \alpha=0^{0}
$$

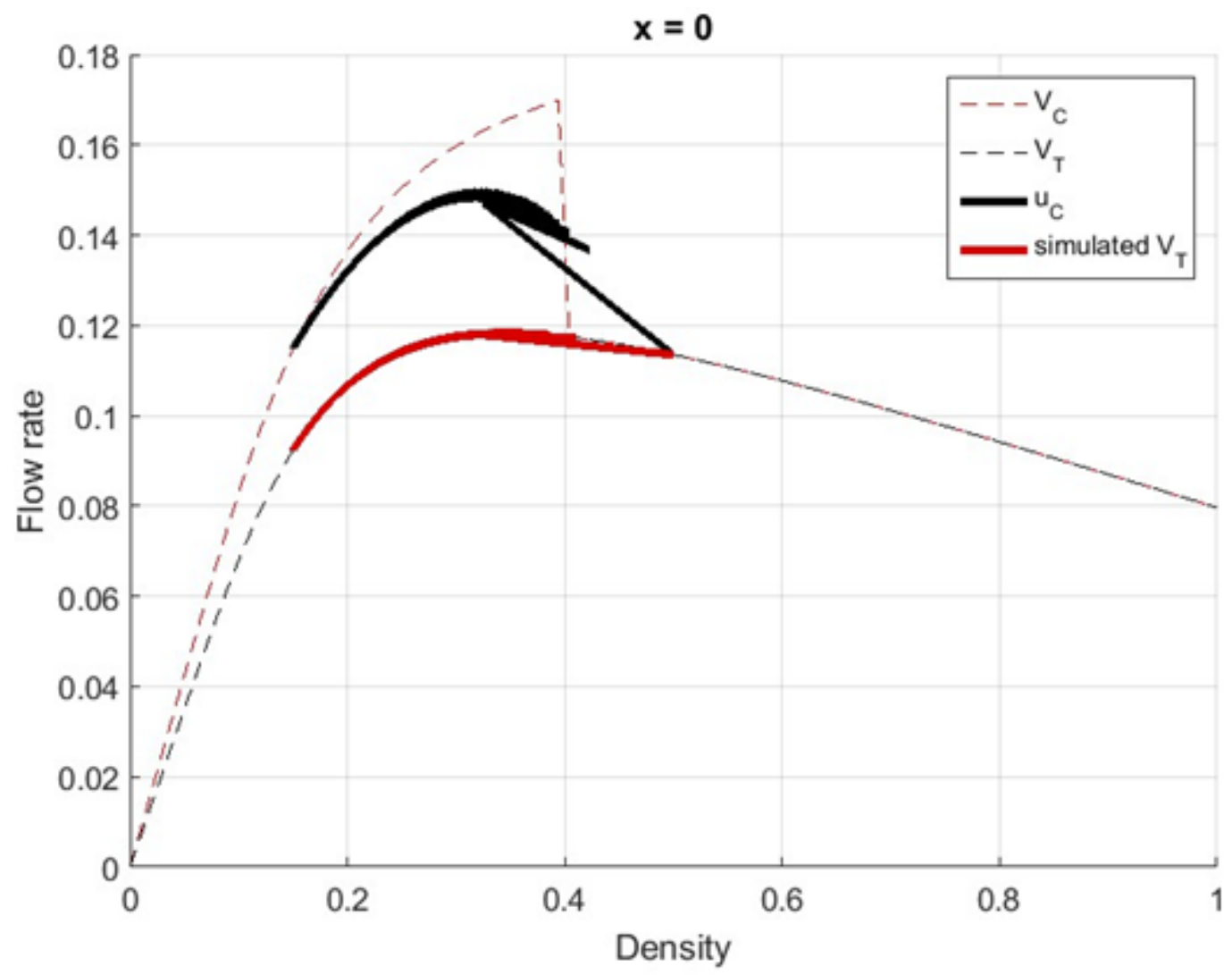

Fig 1:- Depiction of Flow rate against density at $\mathrm{x}=0$, and $\alpha=0$

In this figure the flow rate is much higher for fast moving vehicles in lesser densities as compared to moving bottleneck, this is because the velocity of cars is much higher as compared to that of the trucks. For $\rho \geq 0.5$ the flow rate of cars converges (syncrhonizes) to that of the moving bottleneck and $u^{c}=V_{T}$, because at higher densities the cars have limited chances for overtaking the slow trucks and hence they adapt to the velocity of the trucks, however the general flow rate is still high. 


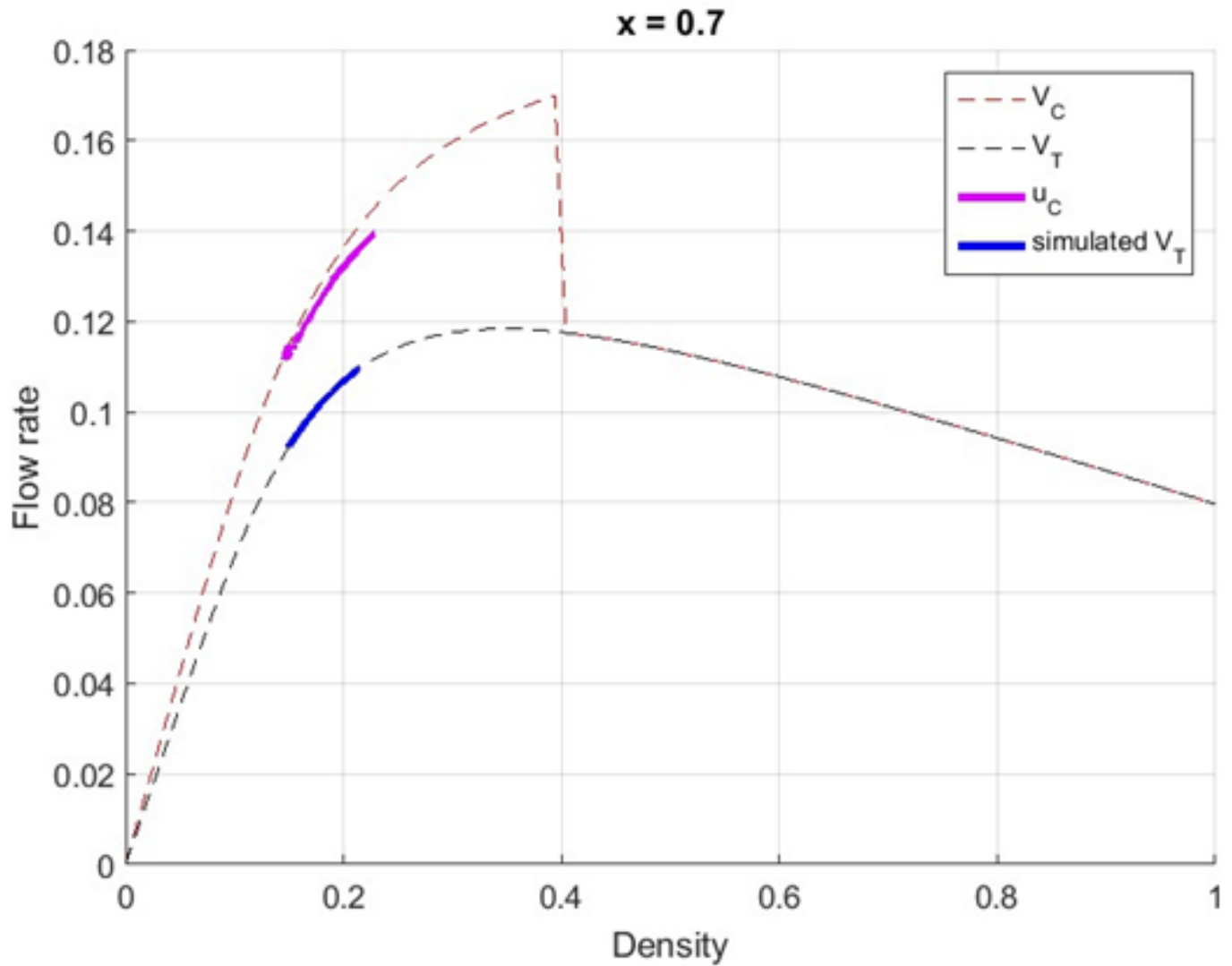

Fig 2:- Depiction of flow rate against Density at $\mathrm{x}=0.7$ and $\alpha=0$

The flow rate is much higher for fast moving cars as compared to that of slow moving trucks. Density of vehicles at $\mathrm{x}=0.7$ has reduced, this is beacuse the fast moving cars are able to overtake the slow moving trucks, as seen from the fundamental diagram the flow rate of the cars and the moving bottleneck are significantly apart. This corresponds to the traffic states transisting back to Free Flow(F).
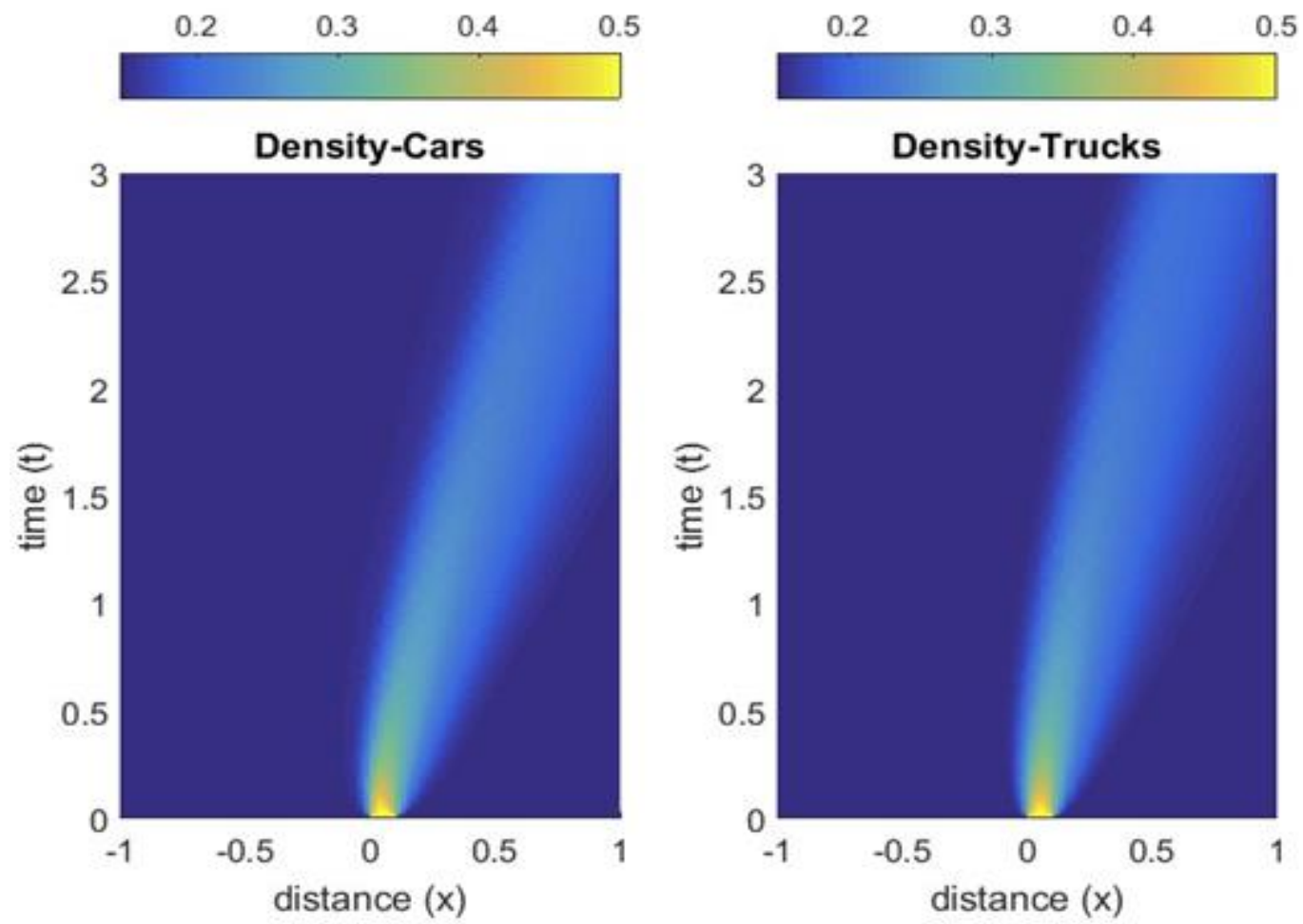

Fig 3:- Depiction of time against distance for density of cars and trucks at $\mathrm{x}=0$ and $\alpha=0$ 
For $-1 \leq \mathrm{x}<0$, which corresponds to free flow, the density of vehicles is very low. This is because it is a free flow regime, where the drivers have more freedom to accelerate to their desired speeds and can also overtake the trucks which have less acceleration capability compared to cars.

At $\mathrm{x}=0$, the density for both cars and trucks is very high. When the density is high, trucks velocity decline, when the cars catch up, they are forced to slow down and adapt the speed of slow moving trucks when they are unable to overtake. This corresponds to traffic transisting from Free Flow (F) to Synchronised Flow (S). For $0<\mathrm{x} \leq 1$, The traffic flow is widened. The density of vehicles is reduced. This is because cars are able to manouver their way and overtake the slow moving trucks. This leads to traffic state transisting from Synchronised Flow $(\mathrm{S})$ to Free Flow $(\mathrm{F})$.
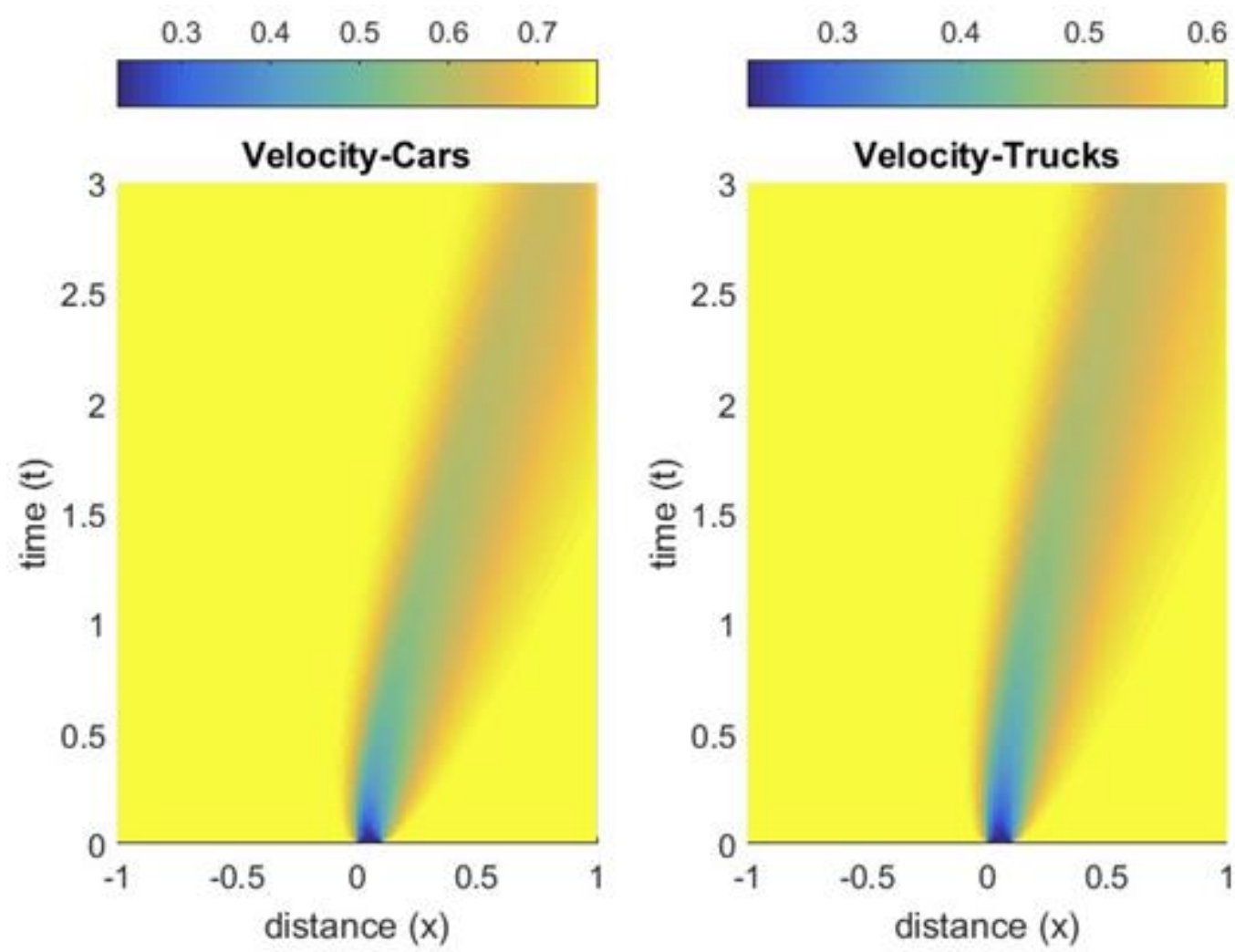

Fig 4:- Depiction of time against distance for velocities of cars and trucks at $\mathrm{x}=0$ and $\alpha=0$.

For $-1 \leq \mathrm{x}<0$, which corresponds to free flow, the velocity of vehicles is very high. This is because the drivers have more freedom to accelerate to their desired speeds and can also overtake the trucks which have less acceleration capability compared to cars. At $\mathrm{x}=0$, the velocity for vehicles is very low. When the density is high, trucks velocity decline, when the cars catch up they are forced to slow down and adapt the speed of slow moving trucks when they are unable to overtake. This corresponds to traffic transisting from Free Flow (F) to Synchronised Flow (S). For $0<\mathrm{x} \leq 1$, the traffic flow is widened. The velocity of vehicles is inceasing gradu- ally. This is because cars are able to manouver their way and overtake the slow moving trucks. This leads to traffic state transisting from Synchronised Flow (S) to Free Flow (F). 


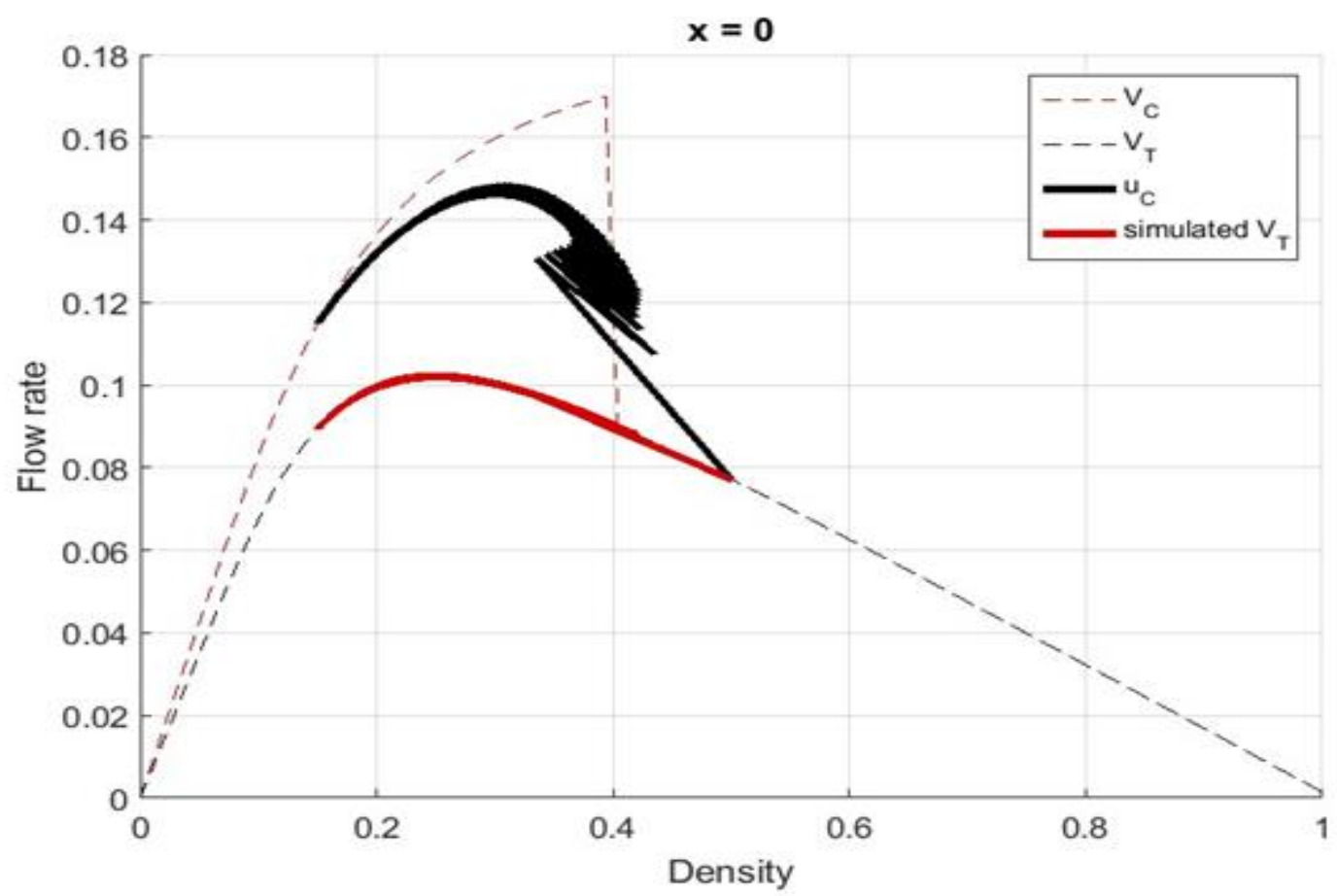

Fig 5:- Depiction of flow rate against density for angle of road inclination, $\alpha=450$ and $\mathrm{x}=0$

The effect of road inclination is shown in figure 5 above via the fundamental dia- gram. In this figure where $\mathrm{x}=0$ marks the beginning of the incline, the flow rate is much higher for fast moving vehicles in lesser densities as compared to moving bottleneck, this is because the velocity of cars is much higher as compared to that of the trucks. As the density increases, at $\rho=0.4$, the fast moving cars tends to overtake, and when they are unable to overtake, at $\rho \geq 0.5$ they are forced to slow down and follow the slow moving trucks hence the flow rate of cars converges (syncrhonizes) to that of the moving bottleneck and uc $=$ VT . This shows traffic Flow Transistion from Free Flow (F) to Synchronsed Flow (S).

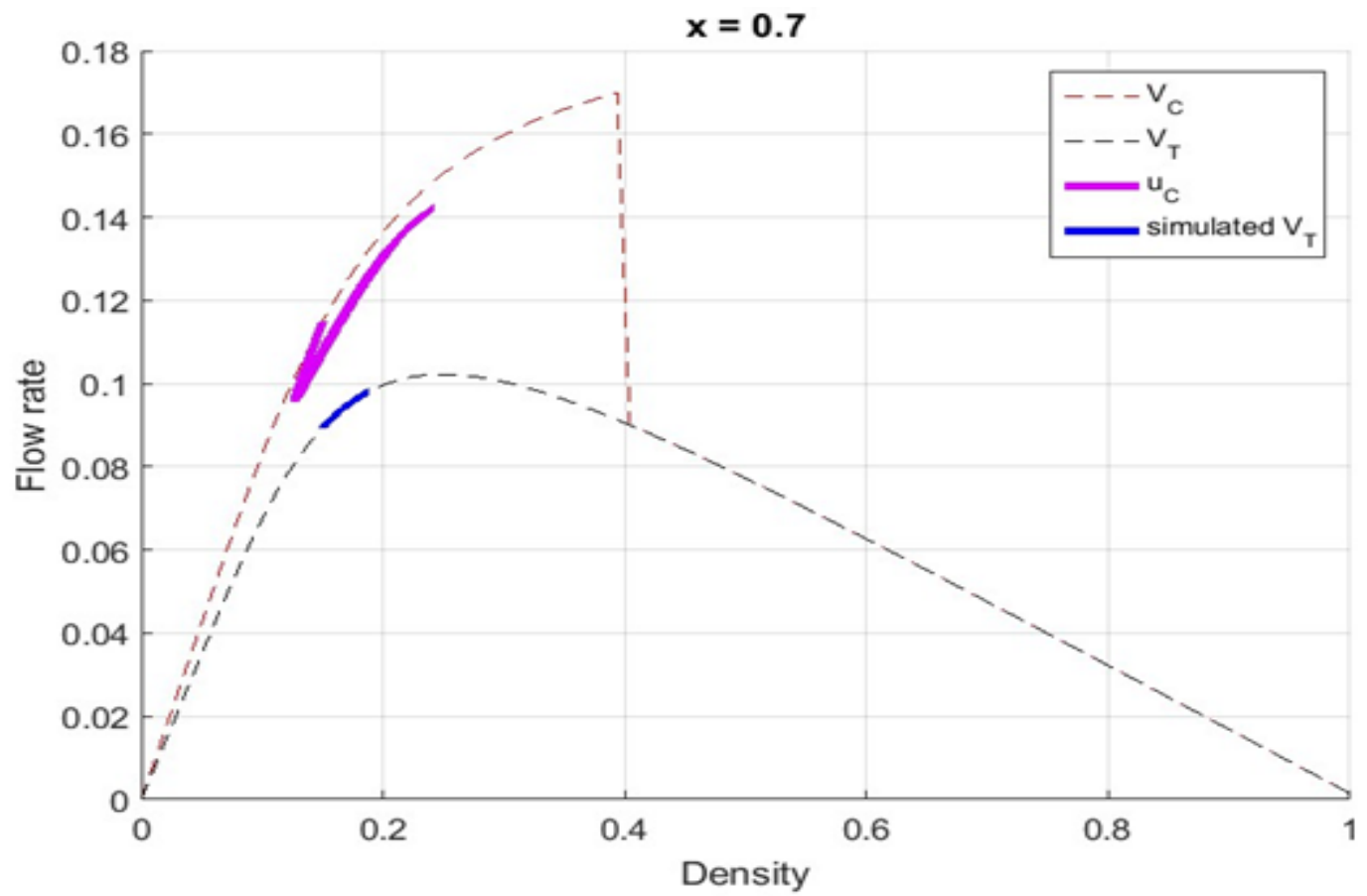

Fig 6:- Depiction of flow rate against density for angle of road inclination, $\alpha=450$ and $\mathrm{x}=0.7$

In this figure, the flow rate of trucks can be seen to decrease as the inclination in- creases. When the angle is high, trucks velocity declines, this is because slow moving trucks have less acceleration ability compared to cars. From the fundamental diagram the flow rate of the cars and the moving bottleneck are significantly apart, this shows that the fast moving cars are able to manouver their way and overtake the slow mov- ing trucks. This marks the Traffic Flow transistion from Synchronised Flow(S) to Free $\operatorname{Flow}(\mathrm{F})$. 

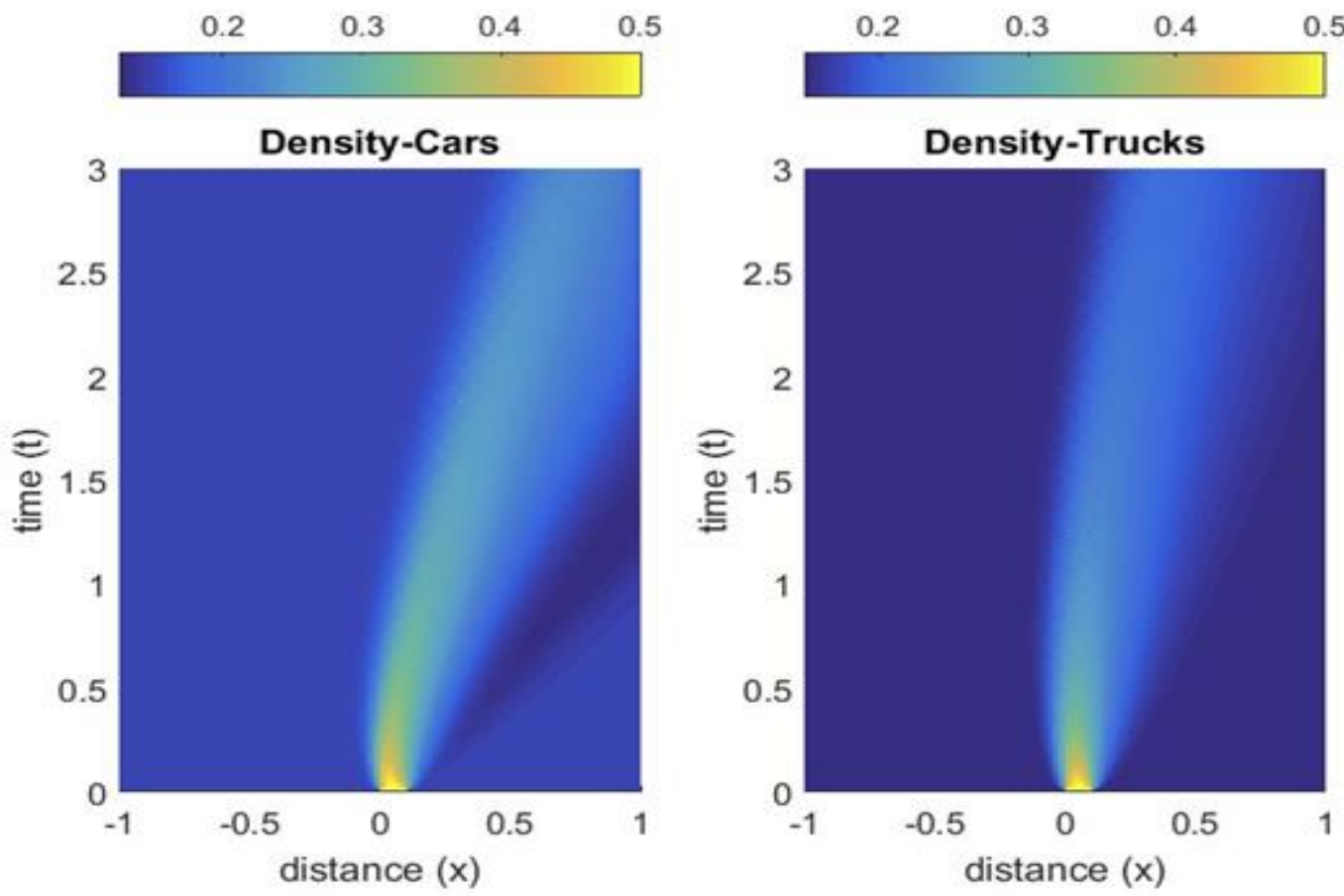

Fig 7:- Depiction of time against distance for density of cars and trucks at $\mathrm{x}=0$ and $\alpha=450$

For $-1 \leq \mathrm{x}<0$, which corresponds to free flow, the density of vehicles is very low. This is because it is a free flow regime, where the drivers have more freedom to accelerate to their desired speeds and can also overtake the trucks which have less acceleration capability compared to cars.

At $\mathrm{x}=0$,this marks the beginning of the incline where the densities of vehicles build up faster than in gentle road. Density for both cars and trucks is very high. When the density is high, trucks velocity decline, when the cars catch up, they are forced to slow down and adapt the speed of slow moving trucks when they are unable to overtake. This corresponds to traffic transisting from Free Flow (F) to Synchronised Flow (S). For $0<\mathrm{x} \leq 1$, The traffic flow is widened. The density of vehicles is reduced. This is because cars are able to manouver their way and overtake the slow moving trucks. This leads to traffic state transisting from Synchronised Flow (S) to Free Flow(F).
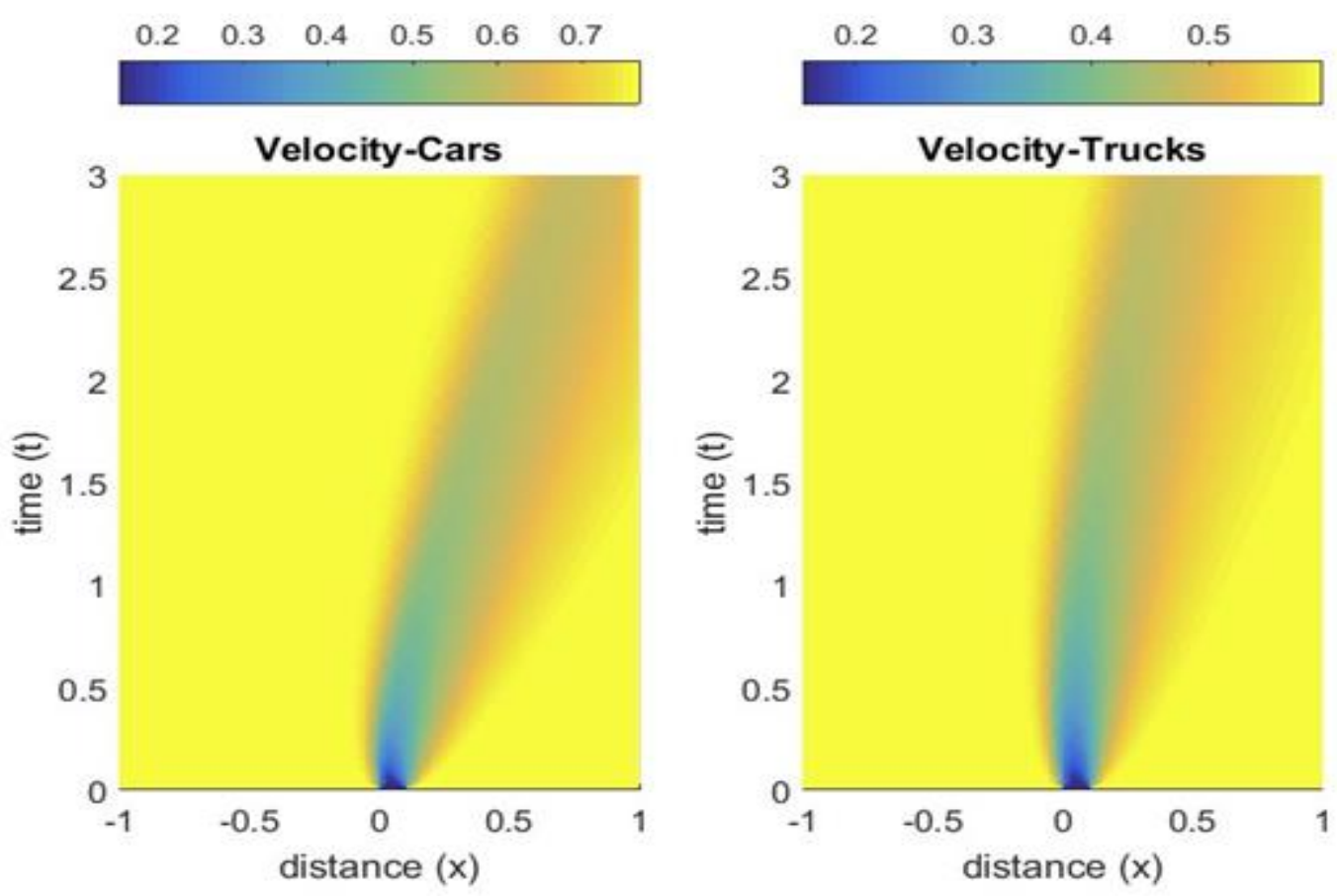

Fig 8:- Depiction of time against distance for velocities of cars and trucks at $\mathrm{x}=0$ and $\alpha=450$ 
For $-1 \leq \mathrm{x}<0$, which corresponds to free flow, the velocity of vehicles is very high. This is because the drivers have more freedom to accelerate to their desired speeds and can also overtake the trucks which have less acceleration capability compared to cars. At $\mathrm{x}=0$, this marks the beginning of the incline. The velocity for vehicles is very low. When the density is high, trucks velocity decline, when the cars catch up, they are forced to slow down and adapt the speed of slow moving trucks when they are unable to overtake. This corresponds to traffic transisting from Free Flow (F) to Synchronised Flow (S).

For $0<\mathrm{x} \leq 1$, The traffic flow is widened. The velocity of vehicles is inceasing grad-ually. This is because cars are able to manouver their way and overtake the slow moving trucks. This leads to traffic state transisting from Synchronised Flow (S) to Free Flow $(\mathrm{F})$.

\section{CONCLUSION}

In this study, macroscopic traffic flow models within the framework of kerner's 3 phase traffic flow theory with consideration of angle of inclination in the context of Kenyan roads have been presented. By construction of the solution to the Riemann problem, set up using the conservative form of the model, the model features have been explored further. The numerical method for solving the macroscopic model in conservative form is discussed and tests are carried out to show the effectiveness of the numerical method used. Using this numerical method we go ahead and simulate traffic flow on a roadway with an incline. Through these simulations, we are to discuss, using Fundamental Di- agram, the Traffic flow transistions i.e F $\rightarrow$ S(Free Flow(F) to Synchronised Flow (S)) and $\mathrm{S} \rightarrow \mathrm{F}$ (Synchronised Flow (S) to Free Flow (F)).

The following conclusions can be drawn:

1. Road inclinations result to moving bottlenecks for mixed traffic flow and these moving bottlenecks trigger a phase transistion from Free Flow to Synchronised Flow.

2. It is reasonable to describe a road inclination through the fundamental diagram.

3. The dependence of the traffic anticipation on the prevailing road conditions brought about by the road inclination and emergence of a moving bottleneck should not be neglected in modelling mixed traffic flow macroscopically.

4. This model will help traffic engineers to improve the existing roads in the con- struction of climbing lanes and consider the best angle of inclination for new construction of uphill road.

\section{REFERENCES}

[1]. Carlos F Daganzo. Requiem for second-order fluid approximations of traffic flow. Transportation Research Part B: Methodological, 29(4):277-286, 1995.

[2]. Paola Goatin. The aw-rascle vehicular traffic flow model with phase transitions. Mathematical and computer modelling, 44(3-4):287-303, 2006.

[3]. Marco Günther, Axel Klar, Thorsten Materne, and Raimund Wegener. An ex- plicitly solvable kinetic model for vehicular traffic and associated macroscopic equations. Mathematical and computer modelling, 35(5-6):591-606, 2002.

[4]. Dirk Helbing and Martin Treiber. Gas-kinetic-based traffic model explaining observed hysteretic phase transition. Physical Review Letters, 81(14):3042, 1998.

[5]. Leif Isaksen and Harold J Payne. Freeway traffic surveillance and control. Pro- ceedings of the IEEE, 61(5):526-536, 1973.

[6]. WL Jin and $\mathrm{H}$ Michael Zhang. The formation and structure of vehicle clusters in the payne-whitham traffic flow model. Transportation Research Part B: Method- ological, 37(3):207-223, 2003.

[7]. Boris S Kerner. Theory of congested highway traffic: empirical features and methods of tracing and prediction. Transportation and Traffic Theory in the 21st Century, pages 417-439, 2002.

[8]. Boris S Kerner. Effect of driver behavior on spatiotemporal congested traffic patterns at highway bottlenecks in the framework of three-phase traffic theory. arXiv preprint arXiv:1012.5159, 2010.

[9]. Mark Erick Kimathi. Mathematical Models for 3PhaseTraffic Flow Theory. PhD thesis, 2012.

[10]. Kazuhito Komada, Shuichi Masukura, and Takashi Nagatani. Effect of gravita- tional force upon traffic flow with gradients. Physica A: Statistical Mechanics and its Applications, 388(14):2880-2894, 2009.

[11]. Michael James Lighthill and Gerald Beresford Whitham. On kinematic waves ii. a theory of traffic flow on long crowded roads. Proceedings of the Royal Society of London. Series A. Mathematical and Physical Sciences, 229(1178):317-345, 1955.

[12]. Bernat Goni Ros, Victor L Knoop, Bart van Arem, and Serge P Hoogendoorn. Empirical analysis of the causes of stop-and-go waves at sags. IET Intelligent Transport Systems, 8(5):499-506, 2014. 